\title{
Symmetry numbers and chemical reaction rates
}

\author{
Antonio Fernández-Ramos • Benjamin A. Ellingson • \\ Rubén Meana-Pañeda · Jorge M. C. Marques · \\ Donald G. Truhlar
}

Received: 14 February 2007 / Accepted: 25 April 2007 / Published online: 11 July 2007

(C) Springer-Verlag 2007

\begin{abstract}
This article shows how to evaluate rotational symmetry numbers for different molecular configurations and how to apply them to transition state theory. In general, the symmetry number is given by the ratio of the reactant and transition state rotational symmetry numbers. However, special care is advised in the evaluation of symmetry numbers in the following situations: (i) if the reaction is symmetric, (ii) if reactants and/or transition states are chiral, (iii) if the reaction has multiple conformers for reactants and/or transition states and, (iv) if there is an internal rotation of part of the molecular system. All these four situations are treated systematically and analyzed in detail in the present article. We also include a large number of examples to clarify some complicated situations, and in the last section we discuss an example involving an achiral diasteroisomer.
\end{abstract}

Keywords Symmetry numbers - Chemical reactions . Transition state theory $\cdot$ Chiral molecules $\cdot$ Multiple conformers $\cdot$ Internal rotation

A. Fernández-Ramos $(\bowtie) \cdot R$. Meana-Pañeda

Departamento de Química Física, Facultade de Química,

Universidade de Santiago de Compostela,

15782 Santiago de Compostela, Spain

e-mail: qftramos@usc.es

B. A. Ellingson · D. G. Truhlar ( $\square)$

Department of Chemistry and Supercomputing Institute, University of Minnesota, 207 Pleasant Street S. E.,

Minneapolis, MN 55455-0431, USA

e-mail: truhlar@umn.edu

J. M. C. Marques

Departamento de Química, Universidade de Coimbra,

3004-535 Coimbra, Portugal

\section{Introduction}

Transition state theory (TST) [1-6] is the most widely used method for calculating rate constants of chemical reactions. The conventional TST rate expression may be written

$k_{\mathrm{TST}}(T)=\sigma \frac{k_{\mathrm{B}} T}{h} \frac{Q_{\mathrm{TS}}(T)}{\Phi_{\mathrm{R}}(T)} \exp \left[-V^{\ddagger} / k_{\mathrm{B}} T\right]$

where $k_{\mathrm{B}}$ is Boltzmann's constant; $h$ is Planck's constant; $V^{\ddagger}$ is the classical barrier height; $T$ is the temperature and $\sigma$ is the reaction-path symmetry number; $Q_{\mathrm{TS}}(T)$ and $\Phi_{\mathrm{R}}(T)$ are the quantum mechanical transition state quasi-partition function and reactant partition function, respectively, without rotational symmetry numbers, and with the zeroes of energy at the zero-point-exclusive energies of the saddle point and equilibrium reactants, respectively. $Q_{\mathrm{TS}}(T)$ is referred to as a quasi-partition function because it is missing the vibrational degree of freedom corresponding to the reaction coordinate. $\Phi_{\mathrm{R}}(T)$ is the unitless reactant partition function for unimolecular reactions and the reactants partition function per unit volume for bimolecular reactions. Specifically for bimolecular reactions $(\mathrm{A}+\mathrm{B} \rightarrow \mathrm{P})$, the reactants partition function can be factorized as $\Phi_{\mathrm{R}}(T)=\Phi_{\text {rel }}^{\mathrm{A}, \mathrm{B}}(T) Q_{\mathrm{R}}(T)=$ $\Phi_{\text {rel }}^{\mathrm{A}, \mathrm{B}}(T) Q_{\mathrm{A}}(T) Q_{\mathrm{B}}(T)$, where $\Phi_{\text {rel }}^{\mathrm{A}, \mathrm{B}}$ is the relative translational motion per unit volume and $Q_{\mathrm{R}}(T)=Q_{\mathrm{A}}(T) Q_{\mathrm{B}}(T)$ is the unitless reactants partition function for the internal motions. The present article is mainly concerned with $\sigma$.

Equation (1) can be generalized by variationally optimizing the transition state (so it is no longer located at the saddle point) and by adding a transmission coefficient to account for recrossing and quantum effects (including tunnelling) [3-6]. These generalizations do not change the considerations involved in giving a value to $\sigma$.

In a classic article, Pollak and Pechukas [7] sorted out conceptual difficulties involving $\sigma$ and proved that it is always 
equal to the ratio of the total symmetry number of the reactant divided by the total symmetry of the transition state. Despite the simplicity and clarity of this result, it can sometimes be confusing to apply it to complex reactions, because it may require considering more than the usual rotational symmetry numbers, and it is discouraging to find that in some recent applications that the symmetry numbers are ignored, ill-defined, or wrong. In addition, the increased complexity of new applications also raises interesting new questions. For these reasons it is useful to review some of the arguments of Pollak and Pechukas and to add some new comments on the subject. It should be noted that symmetry number arguments similar to those of Pollak and Pechukas were also given by Coulson [8].

In conventional TST, the forward thermal rate constant can be calculated with information about only two configurations, i.e., reactants and the transition state. Leaving the symmetry numbers out of the rotational partition function in Eq. (1) means that we are treating the identical particles as distinguishable, and in this case the rotational partition functions would be given by

$Q_{\text {rot }}^{*}(T)=\frac{2 I}{\hbar^{2} \beta}$

for a lineal molecule, where $I$ is the moment of inertia, $\hbar$ is Planck's constant divided by $2 \pi$, and $\beta$ is defined as $1 / k_{\mathrm{B}} T$. For nonlinear molecules, the expression for the rotational partition function of distinguishable particles $Q_{\mathrm{rot}}^{*}(T)$ is

$Q_{\mathrm{rot}}^{*}(T)=\left[\left(\frac{2}{\hbar^{2} \beta}\right)^{3} \pi I_{\mathrm{A}} I_{\mathrm{B}} I_{\mathrm{C}}\right]^{1 / 2}$

where $I_{\mathrm{A}}, I_{\mathrm{B}}$ and $I_{\mathrm{C}}$ are the principal moments of inertia. The symmetry number in Eq. (1) arises from the indistinguishability of identical particles, i.e., the rotational partition is given by

$Q_{\text {rot }}(T)=\frac{1}{\sigma_{\text {rot }}} Q_{\text {rot }}^{*}(T)$

where $\sigma_{\text {rot }}$ is the rotational symmetry number. Therefore the ratio between the rotational partition functions of the transition state, $Q_{\text {rot,TS }}(T)$, and reactants, $Q_{\text {rot, } \mathrm{R}}(T)$, leads to:

$\frac{Q_{\mathrm{rot}, \mathrm{TS}}(T)}{Q_{\mathrm{rot}, \mathrm{R}}(T)}=\frac{\sigma_{\mathrm{rot}, \mathrm{R}}}{\sigma_{\mathrm{rot}, \mathrm{TS}}} \frac{Q_{\mathrm{rot}, \mathrm{TS}}^{*}(T)}{Q_{\mathrm{rot}, \mathrm{R}}^{*}(T)}=\sigma \frac{Q_{\mathrm{rot}, \mathrm{TS}}^{*}(T)}{Q_{\mathrm{rot}, \mathrm{R}}^{*}(T)}$

Therefore, in simple cases, the rotational symmetry numbers in the above partition functions account for all the effects of nuclear indistinguishablility on reaction rates, and the symmetry number in Eq. (1) is given by

$\sigma=\sigma_{\text {rot }, \mathrm{R}} / \sigma_{\text {rot }, \mathrm{TS}}$,

with $\sigma_{\text {rot, } \mathrm{R}}$ and $\sigma_{\text {rot, TS }}$ being the rotational symmetry numbers of the reactants and the transition state, respectively. For a bimolecular reaction, where the reactants are different, $\sigma_{\text {rot, } \mathrm{R}}$
Table 1 Ratios of approximate rotational partition functions for ${ }^{16} \mathrm{O}_{2}$ to the accurate one with only odd $J$

\begin{tabular}{llc}
\hline Temperature (K) & $\begin{array}{l}\text { Classical with } \\
\text { symmetry factor }\end{array}$ & $\begin{array}{l}\text { Quantal without } \\
\text { symmetry }^{\mathrm{a}}\end{array}$ \\
\hline 1,000 & 0.9993 & 2.0000 \\
600 & 0.9988 & 2.0000 \\
300 & 0.9977 & 2.0000 \\
200 & 0.9965 & 2.0000 \\
100 & 0.9931 & 2.0000 \\
50 & 0.9862 & 2.0000 \\
25 & 0.9726 & 2.0000 \\
10 & 0.9326 & 2.0002 \\
5 & 0.8883 & 2.0452 \\
1 & 5.1368 & 22.3732 \\
\hline
\end{tabular}

For this illustration we use the rigid rotor approximation with a moment of inertia of $75,894 \mathrm{~m}_{\mathrm{e}}$ bohr $^{2}$, where $\mathrm{m}_{e}$ is the mass of an electron

${ }^{\text {a }}$ Ratio of Eq. (3a) with $\sigma_{\text {rot }}=2$ to accurate result

b Ratio of partition function with all $J$ to partition function with only odd $J$

is the product of the two $\sigma_{\text {rot }}$ numbers. In some cases, it will not be sufficient to consider just rotational symmetry numbers, $\sigma_{\text {rot }}$. We will need to consider rotational translational symmetry numbers $\sigma_{r-t}$.

It is useful to review the fundamental origin of the symmetry factors. Consider ${ }^{16} \mathrm{O}_{2}$ as an example. Because the ${ }^{16} \mathrm{O}$ nucleus is a boson, the nuclear spin wave function is symmetric under interchange of the nuclei. By Bose-Einstein statistics, the overall wave function must be symmetric under such interchange. Since the nuclear spin wave function is symmetric, and the ground electronic state $\left({ }^{3} \Sigma_{g}^{-}\right)$is odd, the rotational wave function must be antisymmetric (odd). Therefore, half of the rotational quantum numbers $J$ (the even ones) are missing [9]. In the classical limit where sums over rotational levels are replaced by an integral [10], half of the levels being missing decreases the rotational partition function by a factor of two. At low temperature where only a few rotational levels are populated, the factor of two is only approximately correct. In practice, the inclusion of the inaccessible $J=0$ state would cause a very large error at very low temperature. This is illustrated in Table 1, which first shows the ratio of the partition function calculated using Eq. (3a) to the accurate one, and then shows the ratio of the hypothetical partition function for all $J$ to the accurate one. The table shows that the symmetry factor is very close to 2 at most temperatures of interest. The classical approximation is so good that one almost always uses it - the main exception being $\mathrm{H}_{2}$ below room temperature.

When the identical nuclei are fermions, the situation is more complicated. $\mathrm{H}_{2}$ provide the classic example. Since it is treated in most statistical mechanics texts [10], we just summarize the result. It turns out that one fourth of the 
nuclear spin states are forbidden for odd $J$ and three fourths are forbidden for even $J$. Averaging over many states again decreases the partition function by a factor of two.

Polyatomic molecules are more complicated, because there can be more than two identical nuclei, because the vibrational wave functions are not all symmetric, and because the rotational wave functions are more complicated. Consider methane $\left(\mathrm{CH}_{4}\right)$ as an example. The total wave function must be antisymmetric with respect to the exchange of both coordinates and spins of the hydrogen nuclei because they are fermions. As discussed in detail elsewhere [11-13], in order to find the correct nuclear degeneracy associated with each rovibrational state, one has to evaluate a direct product between the permutation group symmetries of the rovibrational states and the nuclear spin wave functions. The net result is that, on average, the molecule has only $1 / 12$ as many states as it would have if the nuclear permutation antisymmetry were not enforced.

In the rest of this article, except briefly in the discussion of the $\mathrm{H}+\mathrm{H}_{2}$ reaction, we shall simply use the classical symmetry factors without considering nuclear spin states or very low temperatures where the classical limit breaks down.

Equation (4) and the product rule given right below it usually suffice, but exceptions arise when reactants in a bimolecular reaction are indistinguishable, when species are chiral, when a reaction is symmetric (sometimes called a degenerate rearrangement), or when TST is used to account for multiple elementary reactions. Continuing along the line of the article of Pollak and Pechukas, it is the objective of this paper to show by examples how to calculate the reaction-path symmetry number of Eq. (1) for any unimolecular or bimolecular reaction.

To evaluate the symmetry number of Eq. (1) the first step is to calculate the rotational symmetry number of the reactants and the transition state. This is explained in Sect. 2. Section 3 illustrates by means of examples how to calculate the symmetry numbers for chemical reactions with additional complications such as chiral isomers, symmetric reactions, low-energy conformers, and internal rotation.

\section{Symmetry numbers and rotation}

Consider the molecules depicted in Structure 1 in their equilibrium configurations: water, ammonia, and the methyl radical. If all of the indistinguishable atoms are labelled and treated as distinguishable and the molecule is fixed in space, the configurations shown in Structure 1 are obtained.

That is, there are two possible configurations for water and six each for nonplanar ammonia and planar methyl radical. In general, the total number of possible configurations equals the $m$ ! permutations of the $m$ equivalent atoms. Of those, we would like to know which of them cannot be transformed into each other by rotation or translation of the whole molecule.

The water molecule, labelled as $\mathbf{a} 2$ in Structure 1, can be transformed into $\mathbf{a} 1$ by a rotation of $180^{\circ}$ and, therefore, there is only one unique configuration. In the case of ammonia $\mathbf{b 5}$ and $\mathbf{b 4}$ are transformed into b1 by clockwise rotations of $120^{\circ}$ and $240^{\circ}$, respectively, whereas $\mathbf{b 3}$ and $\mathbf{b 6}$ can be transformed into b2. However, it is not possible to find any combination of rotation and translation of the ammonia molecule that transforms $\mathbf{b} \mathbf{1}$ into $\mathbf{b} \mathbf{2}$ and, therefore, these two structures are each unique configurations. For the methyl radical $\mathbf{c 2}, \mathbf{c 3}$ and $\mathbf{c 6}$ can be transformed into $\mathbf{c 1}$ by rotations of 180 degrees around the $\mathrm{C}-\mathrm{H}_{\mathrm{a}}, \mathrm{C}-\mathrm{H}_{\mathrm{c}}$ and $\mathrm{C}-\mathrm{H}_{\mathrm{b}}$ axes, respectively; additionally $\mathbf{c 4}$ and $\mathbf{c 5}$ are identical to $\mathbf{c} 1$ by rotations of $120^{\circ}$ and $240^{\circ}$ about an axis that is perpendicular to the molecule and that passes through the central atom. Therefore, there is only one unique configuration of the methyl radical. The rotational symmetry number is given by the number of permutations of $m$ atoms of the same type divided by the number of unique configurations, $n_{\mathrm{d}}$, that remain different under rotation of the molecule, i.e.,

$\sigma_{\text {rot }}=\frac{m !}{n_{\mathrm{d}}}$

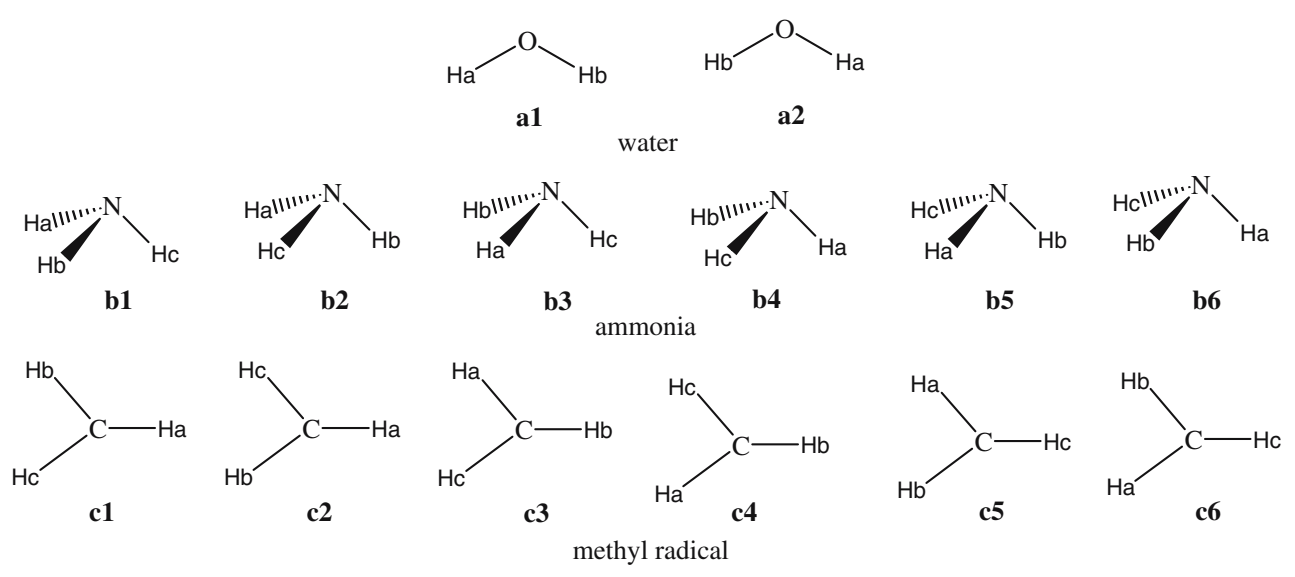

Structure 1 
From these examples we find that for water, ammonia, and the methyl radical the symmetry number is two, three and six, respectively. The symmetry number is uniquely determined by the point group symmetry of the molecule. A molecule without any symmetry (denoted as $C_{1}$ ) has a rotational symmetry number of one. The water molecule with $C_{2 \mathrm{v}}$ point group symmetry in the equilibrium configuration has a rotational symmetry number of two because of the $C_{2}$ and $E$ symmetry operations. The methyl radical with $D_{3 \mathrm{~h}}$ point group symmetry has a rotational symmetry number of 6 because of the identity operation, $E$, three $C_{2}$ rotation axes along any of the $\mathrm{C}-\mathrm{H}$ bonds, and $C_{3}$ (rotation of $120^{\circ}$ ) and $C_{3}^{2}$ (rotation of $240^{\circ}$ ) operations. This makes a total of six rotational symmetry operations. In the same way ammonia, which has $C_{3 \mathrm{v}}$ symmetry, has a symmetry number of three. Therefore, as a general rule, the symmetry number of rotation for a given molecule equals the number of rotational symmetry operations. As a final example let us consider ferrocene, a molecule with $D_{5 \mathrm{~h}}$ point group symmetry. For this molecule the symmetry number of rotation is ten $\left(\mathrm{E}+2 C_{5}+2 C_{5}^{2}+5 C_{2}^{\prime}\right)$.

As stated above, the symmetry number of reactants for a bimolecular reaction ( $\mathrm{A}+\mathrm{B} \rightarrow$ Products) is usually given by the product $\sigma_{\text {rot, } \mathrm{R}}=\sigma_{\text {rot, } \mathrm{A}} \sigma_{\text {rot,B }}$, with $\sigma_{\text {rot, } \mathrm{A}}$ and $\sigma_{\text {rot,B }}$ being the rotation symmetry numbers of $\mathrm{A}$ and $\mathrm{B}$, respectively. However, a bimolecular reaction of the type $\mathrm{A}+\mathrm{A} \rightarrow$ Products constitutes a special case. In this case the symmetry number of reactants is given by $\sigma_{\mathrm{r}-\mathrm{t}, \mathrm{R}}=2 \sigma_{\mathrm{A}}^{2}$. The factor of two appears because of the ability of the reactants to exchange positions between molecules by translation. For instance, let's consider the reaction of a water molecule reacting with another water molecule. The number of possible configurations with labelled hydrogen and oxygen is $2 ! 4$ ! $=48$. Of those, only the 12 depicted in Structure 2 cannot be superimposed by rotation, but only six configurations are
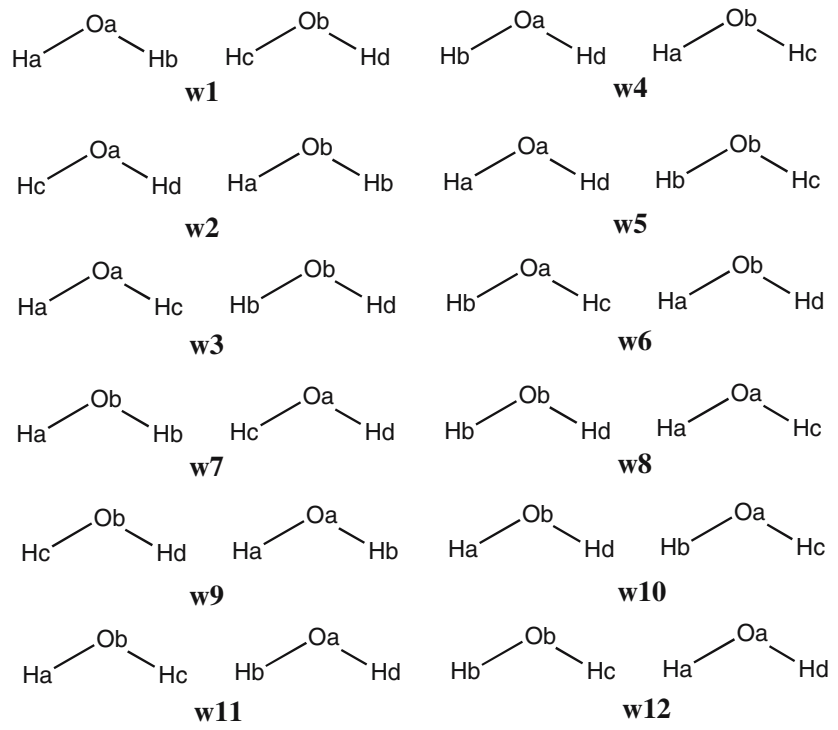

Structure 2

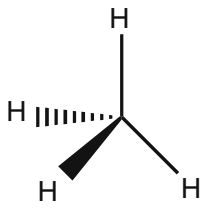

\section{Structure 3}

really distinguishable, because $\mathbf{w 1}=\mathbf{w 9}, \mathbf{w} 2=\mathbf{w} 7, \mathbf{w 3}=\mathbf{w 8}$, $\mathbf{w 4}=\mathbf{w 1 1}$, w5 $=\mathbf{w 1 2}$, and $\mathbf{w 6}=\mathbf{w 1 0}$ by simply translating the first water molecule to the place of the second and vice versa. Therefore, symmetry number is $48 / 6=8$. In this particular case we talk about a symmetry number of rotation-translation $\sigma_{\mathrm{r}-\mathrm{t}, \mathrm{R}}$ which is given by $\sigma_{\mathrm{r}-\mathrm{t}, \mathrm{R}}=2 \sigma_{\text {rot, } \mathrm{A}}^{2}$, which in this case is $\sigma_{\mathrm{r}-\mathrm{t}, \mathrm{R}}=2(2)^{2}=8$.

In some cases the symmetry number of the molecule has a counterintuitive value. For example, consider the molecule of methane, which is depicted in Structure 3. There are four equivalent hydrogen atoms; however, the rotational symmetry number is not four. For methane the number of possible configurations is $4 !=24$ of which only two cannot be superimposed by rotation as indicated below in Structure 4. Thus, the symmetry number is $24 / 2=12$. Again this agrees with what is inferred from the point group symmetry of the molecule, which is $T_{\mathrm{d}}$, because $T_{\mathrm{d}}$ has 12 rotational symmetry operations $\left(E+8 C_{3}+3 C_{2}\right)$.
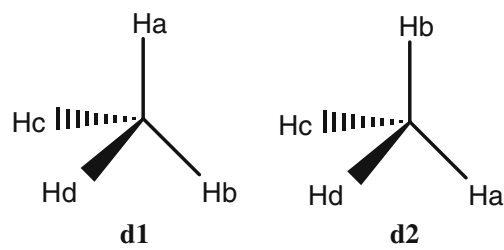

\section{Structure 4}

In other cases the symmetry number corresponds to the number of equivalent atoms, such as the symmetry number for ethane ( $D_{3 \mathrm{~d}}$ symmetry), which has six equivalent hydrogen atoms and also have a rotational symmetry number of six. Table 2 lists the rotation symmetry number for the most common point groups.

\section{The symmetry number in chemical reactions}

Below we illustrate how to calculate the symmetry numbers for a given chemical reaction. This section involves the rotational symmetry numbers of Sect. 2.

\subsection{Some easy examples}

Structure 5 illustrates the bimolecular abstraction of hydrogen from methane by a hydrogen atom.

The symmetry number for the forward reaction, $\sigma_{\mathrm{f}}$, making use of Table 2 and Eq. (4), is given by 


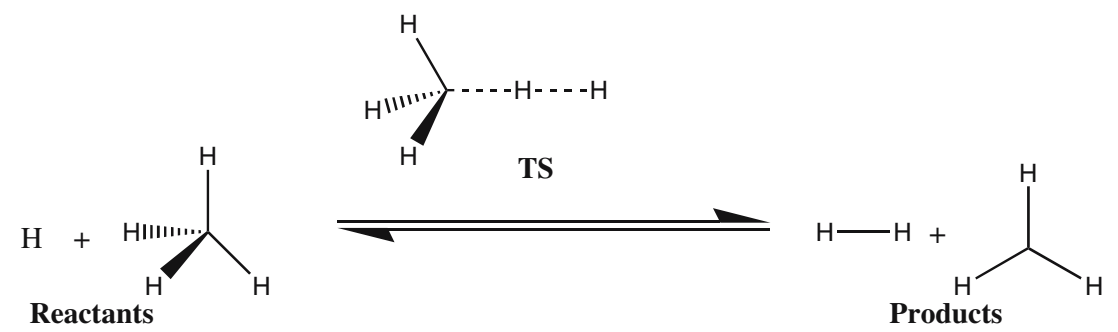

\section{Structure 5}

Table 2 Rotational symmetry number, $\sigma_{\text {rot }}$, of the most common point groups of symmetry

\begin{tabular}{|c|c|c|}
\hline \multirow[t]{13}{*}{ mon point groups of symmetry } & $\mathrm{C}_{1}$ & 1 \\
\hline & $\mathrm{C}_{\mathrm{s}}$ & 1 \\
\hline & $\mathrm{C}_{2}$ & 2 \\
\hline & $\mathrm{C}_{2 \mathrm{v}}$ & 2 \\
\hline & $\mathrm{C}_{3 \mathrm{v}}$ & 3 \\
\hline & $\mathrm{C}_{\infty \mathrm{v}}$ & 1 \\
\hline & $\mathrm{D}_{2 \mathrm{~h}}$ & 4 \\
\hline & $\mathrm{D}_{3 \mathrm{~h}}$ & 6 \\
\hline & $\mathrm{D}_{5 \mathrm{~h}}$ & 10 \\
\hline & $\mathrm{D}_{\infty \mathrm{h}}$ & 2 \\
\hline & $\mathrm{D}_{3 \mathrm{~d}}$ & 6 \\
\hline & $\mathrm{T}_{\mathrm{d}}$ & 12 \\
\hline & $\mathrm{O}_{\mathrm{h}}$ & 24 \\
\hline
\end{tabular}

$\sigma_{\mathrm{f}}=\frac{\sigma_{\mathrm{rot}, \mathrm{R}}}{\sigma_{\mathrm{rot}, \mathrm{TS}}}=\frac{\sigma_{\mathrm{H}} \sigma_{\mathrm{rot}, \mathrm{CH}_{4}\left(T_{\mathrm{d}}\right)}}{\sigma_{\mathrm{rot}, \mathrm{TS}\left(C_{3 \mathrm{v}}\right)}}=\frac{1 \times 12}{3}=4$

which matches the intuitive answer because there are four equivalent hydrogen atoms to abstract. However, sometimes the symmetry number for a reaction is not intuitive. If the hydrogen is abstracted by the $\mathrm{CF}_{3}$ radical (which has $C_{3 \mathrm{v}}$ symmetry in its equilibrium configuration), then the symmetry number is 12 because

$\sigma_{\mathrm{f}}=\frac{\sigma_{\mathrm{rot}, \mathrm{R}}}{\sigma_{\mathrm{rot}, \mathrm{TS}}}=\frac{\sigma_{\mathrm{rot}, \mathrm{CF}_{3}\left(C_{3 \mathrm{v}}\right)} \sigma_{\mathrm{rot}, \mathrm{CH}_{4}\left(\mathrm{~T}_{\mathrm{d}}\right)}}{\left.\sigma_{\mathrm{rot}, \mathrm{TS}\left(C_{3 \mathrm{v}}\right)}\right)}=\frac{3 \times 12}{3}=12$

This can be difficult to comprehend if the symmetry numbers are visualized in terms of the number of equivalent reactions, because in classical mechanics there are four different hydrogen atoms to abstract. However, when it is recognized that there is only one transition state that is quantum mechanically distinguishable, it becomes clear that the symmetry number of 12 arises entirely from the effect that symmetry has on the rotational partition functions of reactants and the transition state. The reader should keep in mind that the symmetry number results from certain rotational states (that would be present for distinguishable particles) being missing in quantum mechanics. It should be noticed that the difference of a factor of three when comparing Eq. (6) with Eq. (7) is unrelated to the internal rotation of the $\mathrm{CH}_{3}$ frag- ment around the $\mathrm{CF}_{3}$ as one may be tempted to think. The relation between internal rotation and symmetry numbers is discussed in Sect. 3.5.

\subsection{Symmetric reactions}

An example of a symmetric reaction is $\mathrm{H}+\mathrm{H}_{2} \rightarrow \mathrm{H}_{2}+\mathrm{H}$. The rotational symmetry numbers for $\mathrm{H}, \mathrm{H}_{2}$, and the linear transition state are one, two, and two, respectively. Therefore, the symmetry number for the reaction is naively expected to be one. However, it is important to be more precise, and ask precisely what observable one is calculating. In fact, the reaction of $\mathrm{H}$ with $\mathrm{H}_{2}$ is not observable as a macroscopic reaction rate. It could, however, be observed by measuring the rate of interconversion of ortho and para hydrogen, and this topic is discussed elsewhere [14]. Similar reactions interconverting the two modifications $[9,15]$ of other molecules, e.g., $\mathrm{N}_{2}$, may also be imagined, although these are not as well known.

The $\mathrm{H}+\mathrm{H}_{2}$ reaction provides one of the rare examples of a case where calculations of the effect of nuclear identicalparticle symmetry have gone beyond the symmetry-number approximation. In particular, Schatz and Kuppermann [16] used the technique of postantisymmetrization [17] to calculate nuclear-motion wave functions with the correct permutation symmetry for $\mathrm{H}+\mathrm{H}_{2}$ with zero total angular momentum. Para-to-ortho rate constants calculated from distinguishableatom rate constants with appropriate classical symmetry numbers differ from those calculated with a proper treatment of permutation symmetry by $0.8,1.6,3,5$, and $26 \%$ at $400,300,250,200$, and $100 \mathrm{~K}$, respectively. Just as nonclassical symmetry effects on rotational partition functions are much smaller for all other molecules than for $\mathrm{H}_{2}$, nonclassical symmetry effects on reaction rates are expected to be much smaller for other reactions than for $\mathrm{H}+\mathrm{H}_{2}$.

In general, any symmetric reaction with just one transition state between reactants and products is unobservable as a macroscopic rate phenomenon, unless one resolves quantum states as in the ortho-para conversion of hydrogen.

\subsection{Chiral species}

In the previous examples none of the species are chiral, so the symmetry number for any nonsymmetric reaction is given 


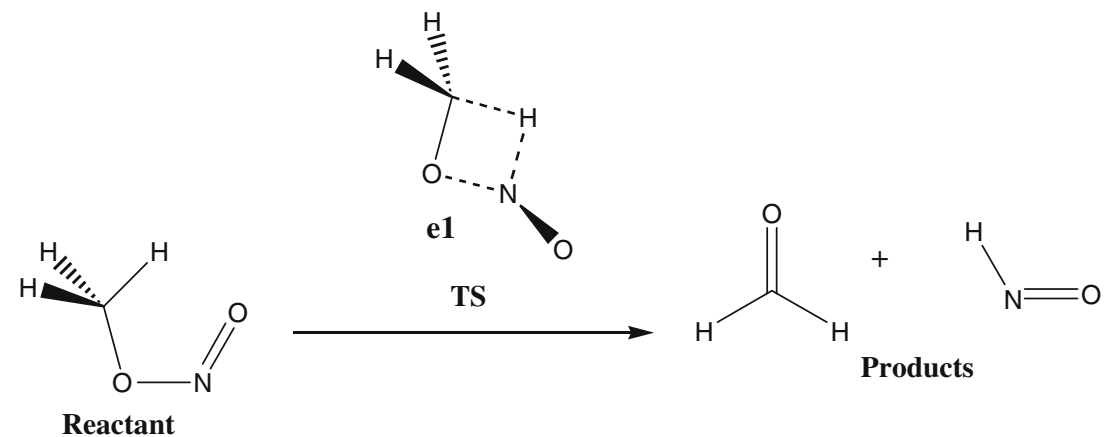

\section{Structure 6}

by Eq. (4). In this section we consider chiral species. We note that some of the species in this section are not considered optically active in the traditional sense because they are short-lived or have low barriers of conversion between the two mirror images. Because of this, we will avoid the words optically active and enantiomer, and instead use the word chi$r a l$, which describes any three-dimensional object that cannot be rotated to coincide with its mirror image. An illustrative example of chiral transition states is the elimination reaction of HNO in the cis-methylnitrite molecule (showed in Structure 6). This elimination reaction has a reactant with $C_{s}$ symmetry in the equilibrium configuration and a four-center transition state with $C_{1}$ symmetry as indicated in Structure 6.

In the transition state of Structure 6 the oxygen atom is not coplanar with the $\mathrm{C}, \mathrm{O}, \mathrm{H}$, and $\mathrm{N}$ atoms. There is another transition state $\mathbf{~} \mathbf{1}^{*}$, with the same energy as $\mathbf{e 1}$, for which the oxygen is on the opposite side the plane as indicated in Structure 7.
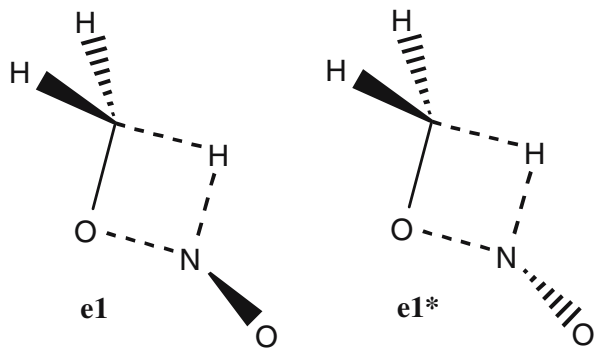

\section{Structure 7}

The structures $\mathbf{e} 1$ and $\mathbf{e} \mathbf{1}^{*}$ are chiral, i.e., they are mirror images that cannot be superimposed by rotation. (It should be noticed that there is no possibility of having chirality when the molecule has one or more planes of symmetry.) There are two separate elementary reactions leading to products from a common reactant, and therefore the total forward rate constant $k_{\mathrm{f}}$ is

$k_{\mathrm{f}}=k_{\mathrm{e} 1}+k_{\mathrm{e} 1^{*}}$

where $k_{\mathbf{e} 1}$ and $k_{\mathbf{e}}{ }^{*}$ are the rate constants for the passage to products from the $\mathbf{e} \mathbf{1}$ and $\mathbf{e} \mathbf{1}^{*}$ transition state structures, respectively. Both rate constants are equal, so the previous equation can be written as

$k_{\mathrm{f}}=2 k_{\mathbf{e} 1}$

Another example of a chiral transition state is the hydrogen abstraction reaction from methanol by a hydrogen atom. The rotational symmetry number for reactants is one. The transition state has $\mathrm{C}_{1}$ symmetry and therefore the symmetry number for the elementary reaction is one. However, Chuang et al. [20] have calculated two possible transition states, f1 and $\mathbf{f} 1^{*}$, which are chiral (see Structure 8 ). As in the previous example, there are two elementary reactions with rate constants that are equal, so the total forward rate constant is given by

$k_{\mathrm{f}}=2 k_{\mathbf{f} 1}$

An example in which the reactants are chiral is the hydrogen abstraction by a hydrogen atom from the $\mathrm{C}(\mathrm{I})(\mathrm{Br})(\mathrm{Cl}) \mathrm{H}$ molecule. This molecule would be present as a racemic mixture of the two possible chiral isomers, as shown in Structure 9 .

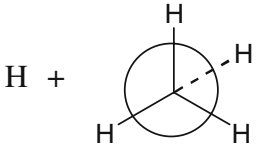

Reactants

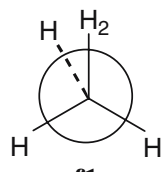

f1

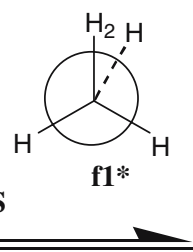

,

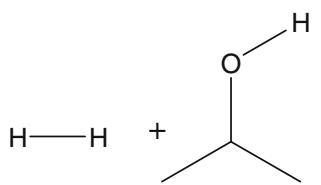

Products

Structure 8 


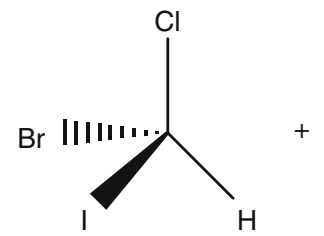

g1

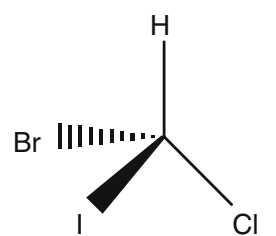

g1*
Structure 9

The hydrogen abstraction reaction from $\mathbf{g} \mathbf{1}$ and $\mathbf{g} 1 *$ would lead to the two chiral transition states shown in Structure 10.

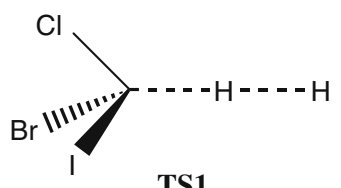

TS1

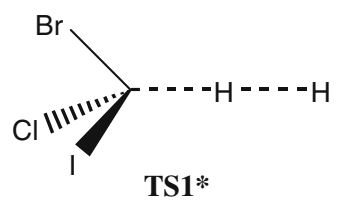

TS1*

\section{Structure 10}

TS1 is only accessible from $\mathbf{g 1}$, and TS1* is only accessible from $\mathbf{g}^{*}$. There are two distinct reactions with unique reactants and transition states that lead to the same products. The rate constant for passage through $\mathbf{T S 1}, k_{\mathbf{T S 1}}(T)$ would be

$k_{\mathbf{T S 1}}(T)=\frac{k_{B} T}{h} \frac{1}{\Phi_{\mathrm{rel}}^{\mathrm{H}, \mathrm{CHClBrI}}(T)} \frac{Q_{\mathbf{T S 1}}(T)}{Q_{\mathbf{g} 1}(T)} \exp \left[-V^{\ddagger} / k_{\mathrm{B}} T\right]$

where $\Phi_{\mathrm{rel}}^{\mathrm{H}, \mathrm{CHClBrI}}(T)$ is the relative translational energy per unit of volume and $Q_{\mathbf{g} \mathbf{1}}(T)$ is the partition function of $\mathbf{g 1}$ excluding translation. In the same way, the rate constant for passage through $\mathbf{T S} 1^{*}$, which can be reached only from $\mathbf{g} \mathbf{1}^{*}$, would be

$k_{\mathbf{T S 1}^{*}}(T)=\frac{k_{B} T}{h} \frac{1}{\Phi_{\mathrm{rel}}^{\mathrm{H}, \mathrm{CHClBrI}}(T)} \frac{Q_{\mathbf{T S 1}^{*}}(T)}{Q_{\mathbf{g} 1^{*}}(T)} \exp \left[-V^{\ddagger} / k_{\mathrm{B}} T\right]$

When using the rate constant to calculate a reaction rate, it is important to recognize what the rate constant refers to. In this example, the overall reaction rate for the formation of products is given by $k_{\mathbf{T S 1}}(T)[\mathbf{g 1}][\mathrm{H}]+k_{\mathbf{T S 1}}(T)\left[\mathbf{g} \mathbf{1}^{*}\right][\mathrm{H}]$, where $[\mathbf{g 1}],\left[\mathbf{g 1}{ }^{*}\right]$ and $[\mathrm{H}]$ are the concentrations of $\mathbf{g 1}, \mathbf{g 1 *}$ and $\mathrm{H}$, respectively. Therefore, after one recognizes the unique elementary reactions, the symmetry number is calculated normally using Eq. (4).

Another example involving a chiral transition state is the hydrogen abstraction reaction from propane by a hydrogen atom. Propane in the equilibrium configuration has $C_{2 \mathrm{v}}$ symmetry and has no optical isomers, so the rotational symmetry is two. In Structure 11, the non-equivalent hydrogen atoms have been labelled as $\mathrm{H}_{\mathrm{a}}, \mathrm{H}_{\mathrm{b}}$ and $\mathrm{H}_{\mathrm{c}}$.

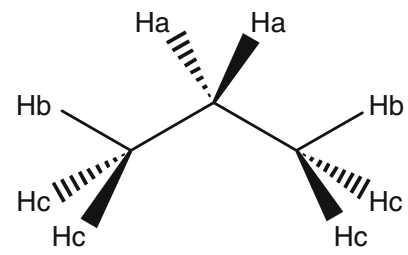

Structure 11

There are eight transition state configurations for the formation of $\mathrm{H}_{2}$, which are depicted in Structure 12. The h1 and h2 transition state configurations correspond to the abstraction of a central hydrogen atom. These configurations are superimposable, with the symmetry number for this single transition state equal to one because the symmetry is $C_{\mathrm{s}}$. The same reasoning can be applied to $\mathbf{h} \mathbf{3}$ and h4. For the last four transition state configurations there is no point group symmetry, but h5 and h8 are superimposable, and so are h6 and h7. However, h5 and h6 cannot be superimposed and therefore are chiral. The thermal rate constant for hydrogen abstraction is the sum of the contributions of all the transition states depicted in Structure 13:

$$
\begin{aligned}
k_{\mathrm{f}}= & \frac{\sigma_{\mathrm{rot}, \mathrm{R}} n_{\mathrm{TS}}(\mathbf{h} \mathbf{1})}{\sigma_{\mathrm{rot}, \mathrm{TS}}(\mathbf{h} \mathbf{1}) n_{\mathrm{R}}} k_{\mathbf{h} \mathbf{1}}+\frac{\sigma_{\mathrm{rot}, \mathrm{R}} n_{\mathrm{TS}}(\mathbf{h} \mathbf{3})}{\sigma_{\mathrm{rot}, \mathrm{TS}}(\mathbf{h} \mathbf{3}) n_{\mathrm{R}}} k_{\mathbf{h} \mathbf{3}} \\
& +\frac{\sigma_{\mathrm{rot}, \mathrm{R}} n_{\mathrm{TS}}(\mathbf{h} \mathbf{5})}{\sigma_{\mathrm{rot}, \mathrm{TS}}(\mathbf{h} \mathbf{5}) n_{\mathrm{R}}} k_{\mathbf{h} \mathbf{5}} \\
= & \frac{2 \times 1}{1 \times 1} k_{\mathbf{h} \mathbf{1}}+\frac{2 \times 1}{1 \times 1} k_{\mathbf{h} \mathbf{3}}+\frac{2 \times 2}{1 \times 1} k_{\mathbf{h} \mathbf{5}} \\
= & 2 k_{\mathbf{h} \mathbf{1}}+2 k_{\mathbf{h} \mathbf{3}}+4 k_{\mathbf{h} \mathbf{5}}
\end{aligned}
$$

where $n_{\mathrm{R}}$ is two for chiral reactants and the unity otherwise, $n_{\mathrm{TS}}(\mathbf{h 5})$ is the number of distinct transition state configurations that can be represented by h5, which is two because h5 and h6 have identical thermodynamic properties, and both $n_{\mathrm{TS}}(\mathbf{h} \mathbf{1})$ and $n_{\mathrm{TS}}(\mathbf{h} \mathbf{3})$ are equal to one because they do not correspond to a chiral transition state. It should be noted that because the transitions states $\mathbf{h 5}$ and $\mathbf{h 6}$ are nonsuperimposable, they are part of different elementary reactions. Doubling the rate constant from two to four for the reaction corresponding to $\mathbf{h 5}$ is simply a shortcut that is utilized by recognizing that mirror images have properties that are equivalent. Calculating each of the four elementary reactions separately would yield the same result achieved in Eq. (13).

The hydrogen abstraction reaction of $\mathrm{OH}$ with propane [18] is calculated in the same manner. For this reaction, the non-degenerate transition states are in Structure 14, and as a first approximation the rate constant is calculated using Eq. (13) Structure 14. However, abstracting with an OH group rather than an $\mathrm{H}$ atom presents a new difficulty because of the ability of the $\mathrm{OH}$ group to rotate. To appropriately model this reaction, each of the transition states needs to be modelled by 


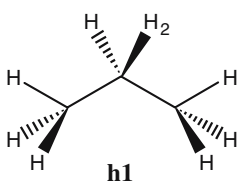

h1

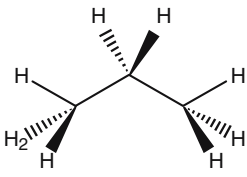

h5

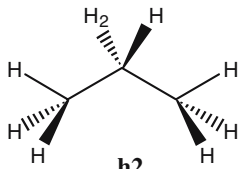

h2

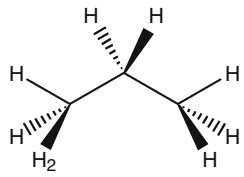

h6

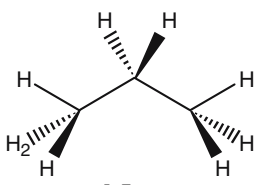

h5<smiles>[1H]C([1H])[14CH3]</smiles>

h3

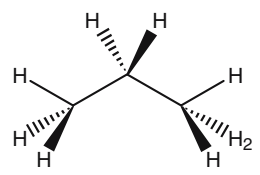

h7

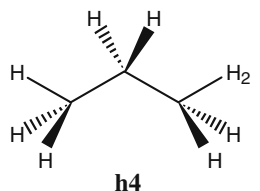

h4

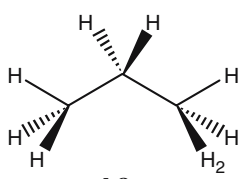

h8

\section{Structure 12}

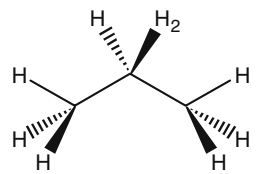

h1

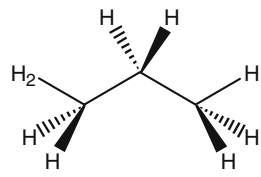

h3

\section{Structure 13}

treating the $\mathrm{OH}$ group as a hindered rotor. This is discussed in Sect. 3.5.

\subsection{Reactions with many conformers}

This section demonstrates how to calculate rate constants for reactions in which the reactants have several conformations. An example is considered where the reactants may be present in two energetically different conformations $\mathbf{j} \mathbf{1}$ and $\mathbf{j} \mathbf{2}$ of which only $\mathbf{j} \mathbf{2}$ can lead to products. A model potential for this type of reaction is depicted in Fig. 1, in which all energies are relative to the most stable conformer of reactants. The reactant conformations are all stable structures that cannot be superimposed by rotation and therefore cannot be taken into account in the rotation symmetry number.

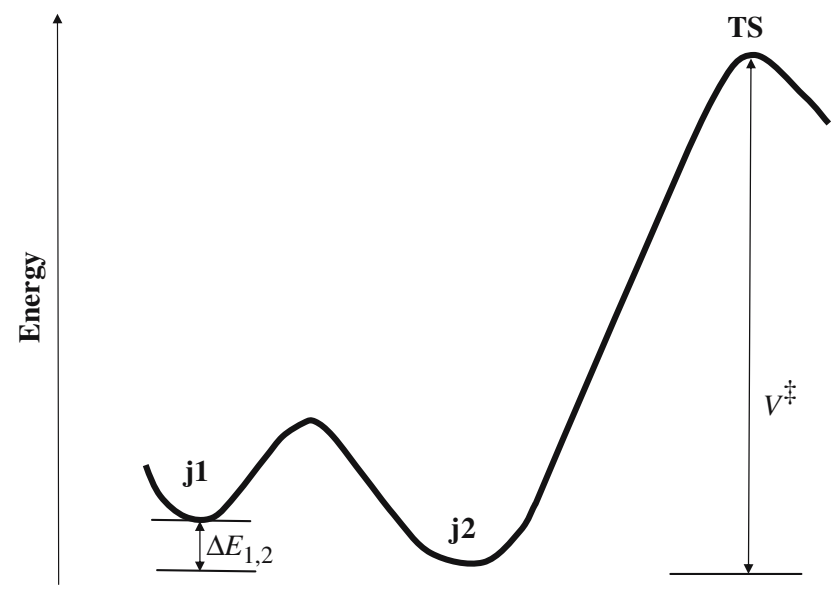

Fig. 1 Schematic illustration showing a model potential for a reaction with a reactant with two different conformations $\mathbf{j} \mathbf{1}$ and $\mathbf{j} \mathbf{2}$, of which only $\mathbf{j} \mathbf{2}$ can lead to products through the transition state TS (see text)
The expression for the forward rate constant would be similar to Eq. (13), with the difference that there are multiple reactants instead of multiple transition states, and they are not necessarily chiral species with the same energy, i.e.

$$
\begin{aligned}
k_{\mathrm{f}}(T)= & \frac{k_{B} T}{h} \frac{Q_{\mathrm{TS}}(T) / \sigma_{\mathrm{rot}, \mathrm{TS}}}{\Phi_{\mathbf{j} 1}(T) e^{-\Delta E_{1,2} / k_{\mathrm{B}} T} / \sigma_{\mathrm{rot}, \mathbf{j} \mathbf{1}}+\Phi_{\mathbf{j} 2}(T) / \sigma_{\mathrm{rot}, \mathbf{j} 2}} \\
& \times \exp \left[-V^{\ddagger} / k_{\mathrm{B}} T\right]
\end{aligned}
$$

where $\sigma_{\text {rot, } \mathbf{j} 1}, \sigma_{\text {rot, } \mathbf{j} 2}$ and $\sigma_{\text {rot,TS }}$ are the rotation symmetry numbers of $\mathbf{j} \mathbf{1}, \mathbf{j} \mathbf{2}$, and the transition state, respectively, $\Delta E_{1,2}$ is the energy difference between $\mathbf{j} \mathbf{1}$ and $\mathbf{j} \mathbf{2}$, i.e., $\Delta E_{1,2}=$ $E(\mathbf{j 1})-E(\mathbf{j} 2)$ and $V^{\ddagger}$ is the barrier height. For the specific case that $\mathbf{j} \mathbf{1}$ and $\mathbf{j} \mathbf{2}$ are nonsuperimposable mirror images of each other, $\sigma_{\text {rot }, \mathbf{j} 1}=\sigma_{\text {rot }, \mathbf{j} \mathbf{2}}, \Delta E_{1,2}=0, \Phi_{\mathbf{j} 1}(T)=\Phi_{\mathbf{j} 2}(T)$ and therefore

$k_{\mathrm{f}}(T)=\sigma \frac{k_{B} T}{h} \frac{Q_{\mathrm{TS}}(T)}{\Phi_{\mathbf{j} 1}(T)} \exp \left[-V^{\ddagger} / k_{\mathrm{B}} T\right]$

where $\sigma$ is given by Eq. (4), but including the factor of two in the denominator due to the chirality of reactants, i.e.,

$\sigma=\frac{\sigma_{\text {rot }, \mathbf{j} 1}}{2 \sigma_{\text {rot }, \mathrm{TS}}}$

For a general reaction with a set of $\left\{\mathrm{R}_{1} \ldots \mathrm{R}_{i} \ldots \mathrm{R}_{\mathrm{N}}\right\}$ conformers of reactants that can lead to a set of $\left\{T_{1} \ldots T_{j} \ldots T_{M}\right\}$ transition states, which lead to products, the forward rate constant is given by

$k_{\mathrm{f}}(T)=\frac{k_{B} T}{h} \frac{\sum_{j=1}^{M} \frac{Q_{\mathrm{T}_{j}}(T) e^{-\Delta E_{\mathrm{T}_{j}} / k_{B} T}}{\sigma_{\mathrm{rot}, \mathrm{T}_{j}}}}{\sum_{i=1}^{N} \frac{\Phi_{\mathrm{R}_{i}}(T) e^{-\Delta E_{\mathrm{R}_{i}} / k_{B} T}}{\sigma_{\mathrm{rot}, \mathrm{R}_{i}}}} \exp \left[-V^{\ddagger} / k_{\mathrm{B}} T\right]$

where $\sigma_{\mathrm{rot}, \mathrm{R}_{i}}, \sigma_{\mathrm{rot}, \mathrm{T}_{j}}, \Phi_{\mathrm{R}_{i}}(T)$ and $Q_{\mathrm{T}_{j}}(T)$ are the rotational symmetry numbers and partition functions of conformations $\mathrm{R}_{i}$ and $\mathrm{T}_{j}$, respectively. The value of $\Delta E_{\mathrm{R}_{i}}$ is a positive energy calculated as the difference between the energy of conformation $\mathrm{R}_{i}$ and the energy of the most stable conformation of reactants. In the same manner, $\Delta E_{\mathrm{T}_{j}}$ is the energy difference between the energy of transition state $\mathrm{T}_{j}$ and the energy of the most stable transition state that leads to products. The value of $V^{\ddagger}$ is calculated as the difference between 


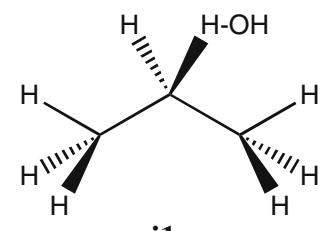

i1

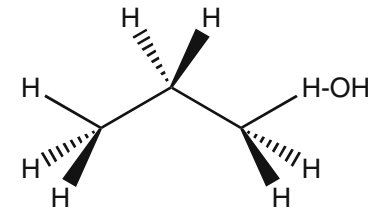

i3

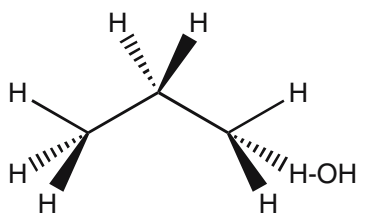

i5

\section{Structure 14}

the energy of the most stable transition state conformation and the energy of the most stable reactant conformation. It should be noticed that the sum also runs over the different chiral isomers and that all the barriers for interconversions of reactants should be smaller than $V^{\ddagger}$. The above expression is valid when the potentials in the vicinity of the stationary points are well described by the harmonic oscillator approximation. Complications may arise from low barriers of conversion between conformations, which may lead to nearly free internal rotations and the breakdown of the harmonic oscillator approximation, which are discussed in Sect. 3.5.

\subsection{Symmetry numbers and internal rotation}

In this section the implications of internal rotation are considered. It should be noticed that rotational symmetry numbers are based on the rotational symmetry of the molecule, i.e., they are based on the rotation of the molecule as a whole, whereas internal rotation involves the rotation of a given part of the molecule relative to the rest of the molecule, and that it is actually a vibrational mode.

Torsion approximations can properly account for indistinguishable minima corresponding to a nearly free internal rotation. This is demonstrated with the example of the hydrogen abstraction reaction from methane by the fluorine atom. For this reaction, some electronic structure calculations predict a transition state with $C_{3 v}$ symmetry (TS1), such as the one shown in Structure 15.

Therefore, the symmetry number for this reaction $\sigma_{\mathrm{f} 1}$ is:

$\sigma_{\mathrm{f} 1}=\frac{\sigma_{\mathrm{rot}, \mathrm{R}}}{\sigma_{\mathrm{rot}, \mathbf{T S} 1}}=\frac{\sigma_{\mathrm{F}} \times \sigma_{\mathrm{rot}, \mathrm{CH}_{4}\left(T_{\mathrm{d}}\right)}}{\sigma_{\mathrm{rot}, \mathbf{T S 1}\left(C_{3 \mathrm{v}}\right)}}=\frac{1 \times 12}{3}=4$

Other electronic structure calculations predict a bent transition state with $C_{S}$ symmetry (TS2) as depicted below

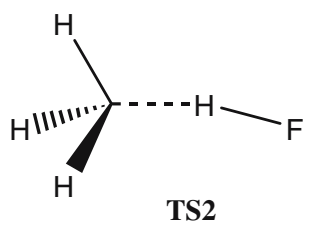

In this case the symmetry number $\sigma_{\mathrm{f} 2}$ is given by

$$
\sigma_{\mathrm{f} 2}=\frac{\sigma_{\mathrm{rot}, \mathrm{R}}}{\sigma_{\mathrm{rot}, \mathbf{T S} 2}}=\frac{\sigma_{\mathrm{F}} \times \sigma_{\mathrm{rot}, \mathrm{CH}_{4}\left(T_{\mathrm{d}}\right)}}{\sigma_{\mathrm{rot}, \mathbf{T S 2}\left(C_{\mathrm{s}}\right)}}=\frac{1 \times 12}{1}=12 .
$$

The symmetry numbers are correct in both cases. The reaction through $\mathbf{T S 1}$ has $C_{3 v}$ symmetry and the reaction through TS2 has $C_{s}$ symmetry, which causes the symmetry number for the reaction through TS2 to be three times larger. However, when the internal rotation for TS2 is treated correctly, the difference in the partition functions at the transition state will largely reconcile the symmetry number difference.

There are three indistinguishable transition state configurations of the type TS2 for each reactive hydrogen, as shown in Structure 16. The three transition state configurations can be obtained from one another through a torsional motion about the $\mathrm{C}-\mathrm{H}$ bond. This internal rotation leads to a potential with three equivalent minima, which coincide with the above configurations, and to three equivalent maxima, which correspond to the eclipsed configurations. The crucial aspects of the potential of internal rotation are the magnitude of both the vibrational frequency of the torsion, $\omega_{\text {tor }}$, and the barrier height between the minima, $\mathrm{W}_{\text {tor }}$.

In the regime of a high torsional frequency and low temperature, i.e., when $k_{B} T<<\hbar \omega_{\text {tor }}$, the potential is well represented by an inverted parabola and the harmonic oscillator $(\mathrm{HO})$ partition function is a good approximation for this torsional mode. Assuming separable $\mathrm{HO}$ partition functions for all the normal modes, the total partition function for TS2 is given by:

$$
\begin{aligned}
Q_{\mathrm{vib}, \mathbf{T S 2}}(T) & =q_{\mathrm{tor}, \mathbf{T S 2}}^{\mathrm{HO}}(T) \prod_{m=1}^{3 N-8} q_{m, \mathrm{vib}, \mathbf{T S 2}}^{\mathrm{HO}}(T) \\
& =\prod_{m=1}^{3 N-7} q_{m, \mathrm{vib}, \mathbf{T S 2}}^{\mathrm{HO}}(T)
\end{aligned}
$$

where $q_{\text {tor, }}^{\mathrm{HO} 2}(T)$ and $q_{m, 3 N-7, \mathbf{T S 2}}(T)$ are two names for the $\mathrm{HO}$ vibrational partition function of the torsional mode with frequency $\omega_{\text {tor }}$, and $q_{m, \mathrm{vib}, \mathbf{T S 2}}^{\mathrm{HO}}(T)$ for $m=1, \ldots, 3 N-8$ are the $\mathrm{HO}$ vibrational partition functions of the other modes. In this case the thermal rate constant evaluated by the TST expression is given by:

$k_{\mathrm{TST}}(T)=12 \frac{k_{B} T}{h} \frac{Q_{\mathrm{rot}, \mathbf{T S 2}}^{*}(T) Q_{\mathrm{vib}, \mathbf{T S} 2}(T)}{\Phi_{\mathrm{R}}(T)} \exp \left[-V^{\ddagger} / k_{\mathrm{B}} T\right]$ 


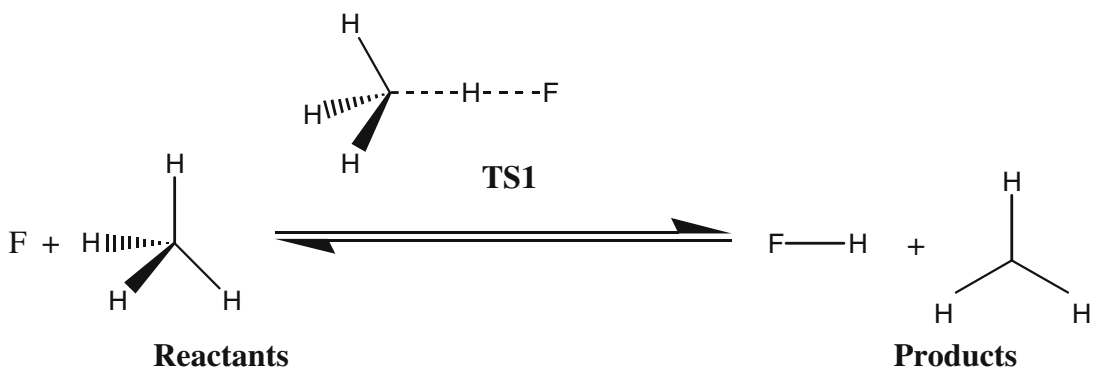

\section{Structure 15}
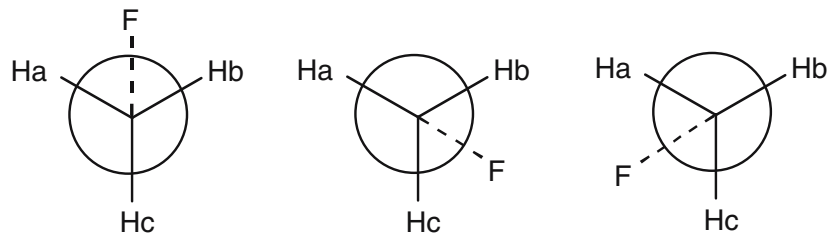

Structure 16

where $Q_{\text {rot,TS2 }}^{*}(T)$ is the $\mathbf{T S 2}$ rotational partition function for distinguishable particles given by Eq. (2b) and $Q_{\text {vib,TS2 }}(T)$ is given by Eq. (20).

However, when $k_{B} T>>W_{\text {tor }}$, the rotation around the $\mathrm{C}-\mathrm{H}$ bond would be nearly free, therefore the classical free rotor (FR) partition function is a good approximation. The FR partition function is given by:

$q^{\mathrm{FR}}(T)=\frac{\left(2 \pi I k_{\mathrm{B}} T\right)^{1 / 2}}{\hbar \sigma_{\text {int }}}=\frac{1}{\sigma_{\text {int }}} q^{\mathrm{FR} *}(T)$

where $I$ is the effective moment of inertia for internal rotation, $q^{\mathrm{FR} *}(T)$ is the FR partition function without the symmetry number for internal rotation, and $\sigma_{\text {int }}$ is the symmetry number for internal rotation. For the above reaction, $\sigma_{\text {int }}$ is three because the three minima depicted in Structure 16 are quantum mechanically indistinguishable. In this case the torsional mode is treated as a free rotation instead of a harmonic vibration, and therefore the TST rate constant is given by:

$$
\begin{aligned}
k_{\mathrm{TST}}(T) & \\
= & 12 \frac{k_{B} T}{h} \frac{Q_{\mathrm{rot}, \mathbf{T S 2}}^{*}(T) \frac{q_{\mathrm{tor}, \mathbf{T S} 2}^{\mathrm{FR}}(T)}{3} \prod_{m=1}^{3 N-8} q_{m, \mathrm{vib}, \mathbf{T S 2}}^{\mathrm{HO}}(T)}{\Phi_{\mathrm{R}}(T)} \\
& \times \exp \left[-V^{\ddagger} / k_{\mathrm{B}} T\right] \\
= & 4 \frac{k_{B} T}{h} \frac{Q_{\mathrm{rot}, \mathbf{T S 2}}^{*}(T) q_{\mathrm{tor}, \mathbf{T S} 2}^{\mathrm{FR}^{*}}(T) \prod_{m=1}^{3 N-8} q_{m, \mathrm{vib}, \mathbf{T S 2}}^{\mathrm{HO}}(T)}{\Phi_{\mathrm{R}}(T)} \\
& \times \exp \left[-V^{\ddagger} k_{\mathrm{B}} T\right]
\end{aligned}
$$

where the symmetry number of rotation is partially cancelled out by the internal rotation symmetry number. This result indicates that a free rotation for the torsional mode is similar to that for which the transition state is of type TS1, since in that case:

$$
\begin{aligned}
k_{\mathrm{TST}}(T)= & 4 \frac{k_{B} T}{h} \\
& \times \frac{Q_{\mathrm{rot}, \mathbf{T S} 1}^{*}(T) q_{m, 3 N-7, \mathbf{T S 1}}^{\mathrm{HO}}(T) \prod_{m=1}^{3 N-8} q_{m, \mathrm{vib}, \mathbf{T S 1}}^{\mathrm{HO}}(T)}{\Phi_{\mathrm{R}}(T)} \\
& \times \exp \left[-V^{\ddagger} / k_{\mathrm{B}} T\right] .
\end{aligned}
$$

The internal rotation for TS2 is very nearly a free rotation, therefore the factor of three that was lost from the rotational partition function when the $C_{3 v}$ symmetry of TS1 was broken reappears in the vibrational partition function from the internal rotation. However, the rate constants for TS1 and TS2 will still be different, as they should be because they are different structures. Most importantly, $q_{\mathrm{tor}, \mathbf{T S 2}}^{\mathrm{FR}}(T)$ may not be similar to $q_{m, 3 N-7, \mathbf{T S 1}}^{\mathrm{HO}}(T)$; additionally the other vibrational and rotational partition functions will likely be slightly different. While the factor of three has been recovered, it depends on how the change in geometry has influenced the partition functions as to whether or not the rate constant for TS1 will be similar to the rate constant for TS2.

It should be noticed that Eq. (21) and Eq. (23) are extreme cases. An important intermediate regime is when $\hbar \omega_{\text {tor }}<<$ $k_{B} T<<W_{\text {tor }}$. The partition function for this intermediate (I) case is given by:

$Q^{\mathrm{I}}(T)=\frac{k_{\mathrm{B}} T}{\hbar \omega_{\text {tor }}}$

For a reaction in which internal rotations may play a role, it would be desirable to have an expression for the partition function that is accurate for the two extreme cases of FR and $\mathrm{HO}$ and displays smooth behaviour for intermediate cases. A function with these characteristics has been given elsewhere [19] and has the form:

$Q=Q^{\mathrm{HO}} \tanh \left(Q^{\mathrm{FR}} / Q^{\mathrm{I}}\right)$

where $Q^{\mathrm{HO}}, Q^{\mathrm{FR}}$ and $Q^{\mathrm{I}}$ are the partition function for the $\mathrm{HO}, \mathrm{FR}$ and intermediate case of Eq. (25), respectively. The example of this section deals with internal rotations in the transition state but the same reasoning can be applied to internal rotations in the reactants or in both reactants and the transition state. 
The $\mathrm{H}+\mathrm{CH}_{3} \mathrm{OH} \rightarrow \mathrm{H}_{2}+\mathrm{CH}_{2} \mathrm{OH}$ abstraction reaction [20] is a case where an internal rotation approximation is able to account for multiple configurations. If two of the three hydrogens of the methyl group were deuterated, the rate constant for hydrogen abstraction is expected to be approximately three times smaller than that involving the undeuterated methanol because there are one third as many reactive atoms. The rate constant of interest is for the $\mathrm{H}+\mathrm{HCD}_{2} \mathrm{OH} \rightarrow$ $\mathrm{H}_{2}+\mathrm{CD}_{2} \mathrm{OH}$ process. The molecule $\mathrm{HCD}_{2} \mathrm{OH}$ has the three conformers depicted in Structure 17, where k1 and k1* are chiral.<smiles>[2H]C12CCC3CC(C)(CC1)C32</smiles>

k1

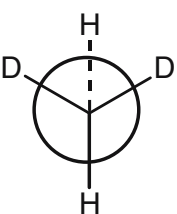

k2

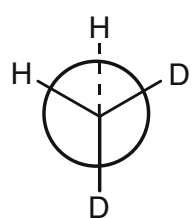

k1*
Structure 17

Structure 18 depicts the two lowest-energy transition states starting from the above reactants

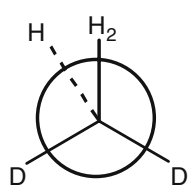

Tk1

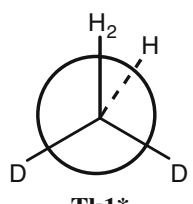

Tk1*

\section{Structure 18}

For this case Eq. (17) leads to the following expression: where $\Phi_{\mathrm{rel}}^{\mathrm{H}, \mathrm{HCD}_{2} \mathrm{OH}}$ is the relative translational partition function per unit of volume as mentioned in the Introduction. Noticed that this rate constant is about one third the rate constant obtained for the undeuterated reaction as it should be. For distinguishable conformers, as is the case here, it may be desirable to use a torsional method that accounts for the multiple wells of the reactant and/or transition state regions instead of using a simple HO approximation. Specifically, the $\mathrm{HO}$ approximation for the torsional mode is replaced by the multiconformer $\mathrm{HO}(\mathrm{MC}-\mathrm{HO})$ treatment: $[21,22]$

$Q_{\mathrm{tor}}^{\mathrm{MC}-\mathrm{HO}}=\sum_{j=1}^{P} \frac{e^{-\beta\left(U_{j}+\hbar \omega_{\mathrm{tor}, j} / 2\right)}}{1-e^{-\beta \hbar \omega_{\mathrm{tor}, j}}}$

where $P$ is the number of distinguishable minima, $\omega_{\mathrm{tor}, j}$ is the harmonic frequency at minimum $j$ of the torsional mode, and $U_{j}$ is the energy of well $j$ of this mode relative to the lowest well of this mode. In this example we have labeled the torsional mode as $3 N-7$ in the transition state and $3 N-$ 8 in reactants In the case of the deuterated methanol, all of the wells have the same energy so $U_{j}=0$ in all cases, but $\mathbf{k} \mathbf{2}$ does have a frequency for the torsional mode that is different than $\mathbf{k} \mathbf{1}$ and $\mathbf{k} \mathbf{1}^{*}$. In the same manner, the MC-HO method is used as part of the torsional method to calculate the partition function for the transition state. However, because the multiple transition state configurations are now accounted for using the torsional method, the symmetry number for the transition state is no longer equal to two, rather it is equal to one. This MC-HO partition function for the torsion is then used in the equations given above and the TST rate constant is given by Eq. (29),

$$
\begin{aligned}
& k_{\mathrm{TST}}(T)=\frac{k_{B} T}{h} \frac{1}{\Phi_{\mathrm{rel}}^{\mathrm{H}, \mathrm{HCD}} \mathrm{OH}_{2}} \exp \left[-V^{\ddagger} / k_{\mathrm{B}} T\right] \frac{Q_{\mathrm{rot}, \mathbf{T k 1}}^{*}(T) Q_{\mathrm{vib}, \mathbf{T k} \mathbf{1}}(T)+Q_{\mathrm{rot}, \mathbf{T k} 1^{*}}^{*}(T) Q_{\mathrm{vib}, \mathbf{T k} 1^{*}}(T)}{Q_{\mathrm{rot}, \mathbf{k} \mathbf{1}}^{*}(T) Q_{\mathrm{vib}, \mathbf{k} \mathbf{1}}(T)+Q_{\mathrm{rot}, \mathbf{k} \mathbf{2}}^{*}(T) Q_{\mathrm{vib}, \mathbf{k} \mathbf{2}}(T)+Q_{\mathrm{rot}, \mathbf{k} 1^{*}}^{*}(T) Q_{\mathrm{vib}, \mathbf{k} 1^{*}}(T)} \\
& =\frac{k_{B} T}{h} \frac{1}{\Phi_{\mathrm{rel}}^{\mathrm{H}, \mathrm{HCD}} \mathrm{OH}_{2}} \frac{2 Q_{\mathrm{rot}, \mathbf{T k} \mathbf{1}}^{*}(T) Q_{\mathrm{vib}, \mathbf{T k} \mathbf{1}}(T)}{2 Q_{\mathrm{rot}, \mathbf{k} \mathbf{1}}^{*}(T) Q_{\mathrm{vib}, \mathbf{k} \mathbf{1}}(T)+Q_{\mathrm{rot}, \mathbf{k} \mathbf{2}}^{*}(T) Q_{\mathrm{vib}, \mathbf{k} \mathbf{2}}(T)} \exp \left[-V^{\ddagger} / k_{\mathrm{B}} T\right]
\end{aligned}
$$

$$
\begin{aligned}
& k_{\mathrm{TST}}(T)=\frac{k_{B} T}{h} \frac{1}{\Phi_{\mathrm{rel}}^{\mathrm{H}, \mathrm{HCD}} \mathrm{OH}^{\mathrm{OH}}} \frac{Q_{3 N-7, \mathrm{TS}}^{\mathrm{MC}-\mathrm{HO}}(T) Q_{\mathrm{rot}, \mathbf{T k 1}}^{*}(T) \prod_{m=1}^{3 N-8} q_{m, \mathbf{v i b}, \mathbf{T k 1} 1}(T)}{Q_{3 N-6, \mathrm{R}}^{\mathrm{MC}-\mathrm{HO}}(T) Q_{\mathrm{rot}, \mathbf{k} \mathbf{1}}^{*}(T) \prod_{m=1}^{3 N-7} q_{m, \mathbf{v i b}, \mathbf{k} \mathbf{1}}(T)} \exp \left[-V^{\ddagger} / k_{\mathrm{B}} T\right] \\
& =\frac{k_{B} T}{h} \frac{1}{\Phi_{\mathrm{rel}}^{\mathrm{H}, \mathrm{HCD}} 2 \mathrm{OH}} \frac{\sum_{j=1}^{2}\left(\frac{e^{-\beta \hbar \omega_{3 N-7, j} / 2}}{1-e^{-\beta \hbar \omega_{3 N-7, j}}}\right) \times Q_{\mathrm{rot}, \mathbf{T k} \mathbf{1}}^{*}(T) \prod_{m=1}^{3 N-8} q_{m, \mathbf{v i b}, \mathbf{T k} \mathbf{1}}(T)}{\sum_{j=1}^{3}\left(\frac{e^{-\beta \hbar \omega_{3 N-6, j} / 2}}{1-e^{-\beta \hbar \omega_{3} N-6, j}}\right) \times Q_{\mathrm{rot}, \mathbf{k} \mathbf{1}}^{*}(T) \prod_{m=1}^{3 N-7} q_{m, \mathrm{vib}, \mathbf{k} \mathbf{1}}(T)} \exp \left[-V^{\ddagger} / k_{\mathrm{B}} T\right]
\end{aligned}
$$

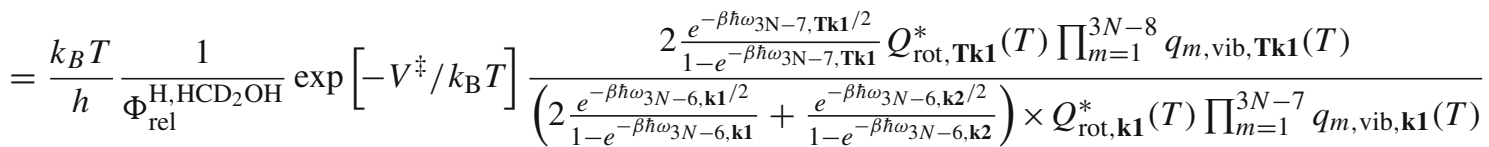



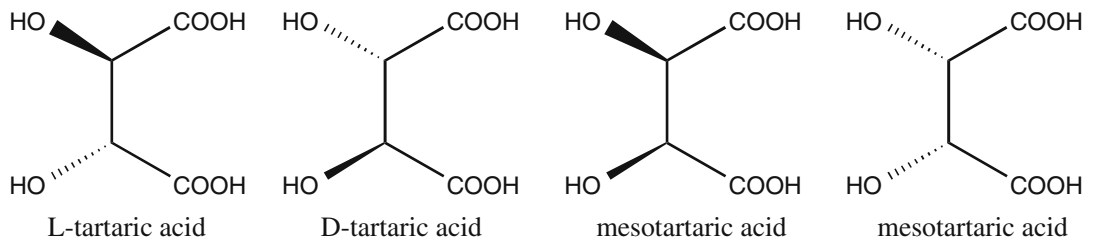

\section{Structure 19}

Because the summation accounts for three degenerate wells, the torsional treatment causes the reactant partition function to be approximately three times as large as the partition function for undeuterated methanol, which causes the rate constant for the deuterated methanol to be about $1 / 3$ as large, just as expected.

While the rate constant for undeuterated methanol was calculated in an earlier example by accounting for the two nonsuperimposable transition states that were low in energy, a better way to account for the entire range of motion is to use a torsional method for the transition state as well. Note that the strategy used for this example is very similar to the strategy discussed in Sect. 3.4. The difference is that in Sect. 3.4, the vibrations were only calculated using the $\mathrm{HO}$ approximation, whereas this example uses the MC-HO partition function in conjunction with Eq. (26). Therefore, as the motion becomes more like a free internal rotation, the rotational partition function calculated by Eq. (26) remains qualitatively correct. If the multiple conformations of the torsion were calculated using the strategy in Sect. 3.4, there would be substantial error in regions where the HO approximation was not valid. The drawback of Eq. (29) is that the rotational and vibrational partition functions, with exception of the torsional mode, are considered to be equal for all conformers.

For further details on the treatment of internal rotation, we refer to references [19-22].

\subsection{An example involving an achiral diasteromer}

Tartaric acid is an organic acid that is naturally occurring in the L-(-)-tartaric acid form [23,24]. Tartaric acid has two carbon stereocenters and which allows it to be synthesized in three distinguishable configurations. There is a set of enantiomers, and there is also a meso complex. These configurations are shown in Structure 19.

While L-tartaric acid and D-tartaric acid are distinguishable, the two drawings of mesotartaric acid are superimposable, so there is only a single distinguishable configuration for mesotartaric acid. That is, there are three stereoisomers of tartaric acid; two of them are enantiomers, and the third is an achiral diastereomer.

For a reaction where the acidic proton is abstracted by $\mathrm{OH}^{-}$to create the conjugate base, we illustrate the transition

states (this time explicitly drawing the carboxyl group on the reacting carbon).

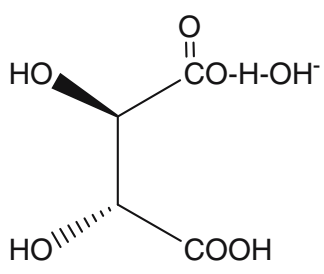

L-TS 1

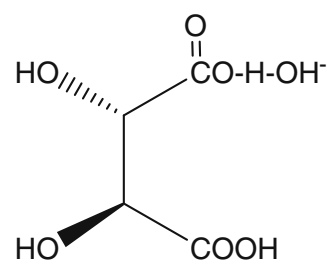

D-TS1

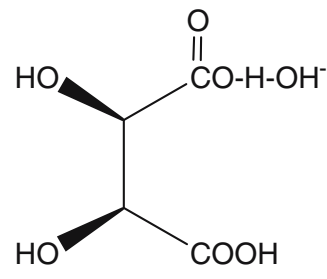

m-TS1

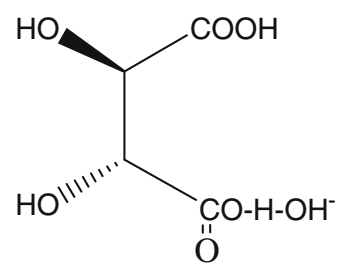

L-TS2

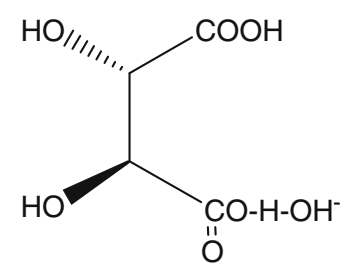

D-TS2

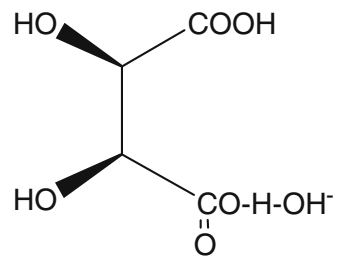

$\mathrm{m}-\mathrm{TS} 2$

\section{Structure 20}

The transition states have been labelled according to which reactant they originated from, where L-indicates that it originated from L-tartaric acid, D-indicates that it originated from $\mathrm{D}$-tartaric acid, and $\mathrm{m}$ - indicated that is originated from mesotartaric acid. The transition states that originate from the meso reactant are labelled with m-instead of meso- because they are not superimposable. L-TS1 and L-TS2 are superimposable by a rotation, so there is only a single indistinguishable transition state for this reaction. This is also true for D-TS1 and D-TS2. For the L- and D-enantiomers, there is a single elementary reaction leading to a single product for each enantiomer, as shown in Structure 21.

The two transition states for mesotartaric acid are not superimposable. There are two elementary reactions with 


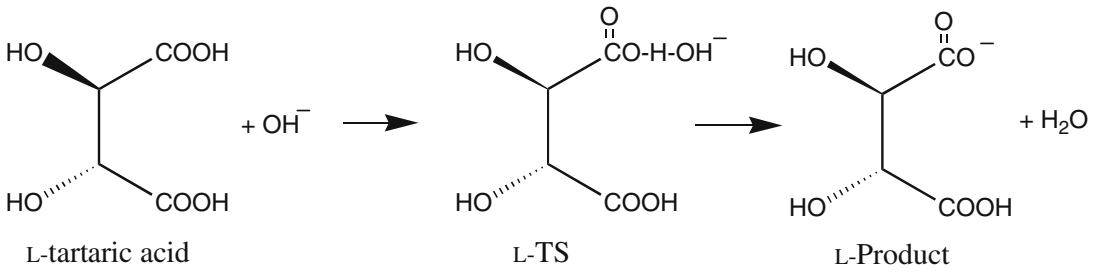

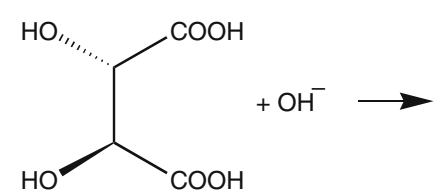

D-tartaric acid

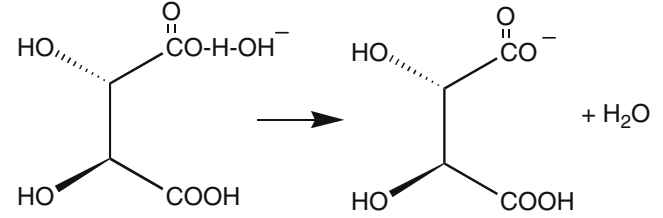

D-TS

D-Product

\section{Structure 21}

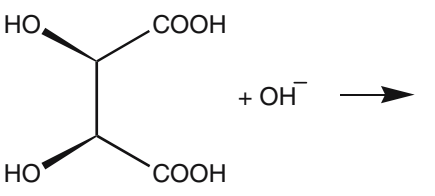

mesotartaric acid<smiles>C[C-][O+]=O</smiles>

mesotartaric acid<smiles>O=C(O)C(O)[C@@H](O)C(=O)O[O-]</smiles>

m-TS1

m-Product1

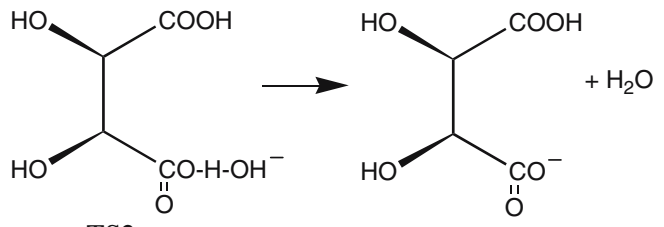

$\mathrm{m}-\mathrm{TS} 2$

m-Product 2

\section{Structure 22}

distinct transition states that lead to distinct products. These are shown in Structure 22.

Now that the elementary reactions have all been determined, the symmetry numbers for the rate constants can be calculated. For L-tartaric acid, the reactant has $C_{2}$ symmetry and the transition state has $C_{1}$ symmetry, which yields a symmetry number of 2 . Therefore, the forward rate constant for the formation of L-product is

$k_{\mathrm{L}}(T)=2 \frac{k_{B} T}{h} \frac{1}{\Phi_{\mathrm{rel}}^{\mathrm{L}-\mathrm{R}, \mathrm{OH}^{-}}} \frac{Q_{\mathrm{L}-\mathrm{TS}}(T)}{Q_{\mathrm{L}-\mathrm{R}}(T) Q_{\mathrm{OH}^{-}}} \exp \left[-V^{\ddagger} / k_{\mathrm{B}} T\right]$

where $Q_{\mathrm{L}-\mathrm{R}}(T)$ is the reactant partition function for L-tartaric acid excluding translation and $\Phi_{\text {rel }}^{\mathrm{L}-\mathrm{R}, \mathrm{OH}^{-}}$is the relative translational partition function per unit volume as stated in Sect. 1. Similarly, the forward rate constant for the formation of D-product is

$$
k_{\mathrm{D}}(T)=2 \frac{k_{B} T}{h} \frac{1}{\Phi_{\mathrm{rel}}^{\mathrm{D}-\mathrm{R}, \mathrm{OH}^{-}}} \frac{Q_{\mathrm{D}-\mathrm{TS}}(T)}{Q_{\mathrm{D}-\mathrm{R}}(T) Q_{\mathrm{OH}^{-}}} \exp \left[-V^{\ddagger} / k_{\mathrm{B}} T\right]
$$

where $Q_{\mathrm{D}-\mathrm{R}}(T)$ is the reactant partition function for D-tartaric acid. At this point, we are able to take advantage of mirror images, and we find that $Q_{\mathrm{L}-\mathrm{TS}}(T)=Q_{\mathrm{D}-\mathrm{TS}}(T)$, $Q_{\mathrm{L}-\mathrm{R}}(T)=Q_{\mathrm{D}-\mathrm{R}}(T)$, and the barrier heights are the same, therefore $k_{\mathrm{L}}(T)=k_{\mathrm{D}}(T)$.

For mesotartaric acid, the reactant has $C_{s}$ symmetry, and both of the transition states have $C_{1}$ symmetry, which yield a rotational symmetry number of 1 . However, the chiral transition states present an additional difficulty, because the single reactant can follow two different elementary reactions, so the symmetry number for the formation of the two products is two. Therefore, each elementary reaction has a symmetry number of one due to the chirality of the transition states [7]. The rate constants for meso-product 1 and meso-product 2 are

$k_{\mathrm{m}-1}(T)=\frac{k_{B} T}{h} \frac{1}{\Phi_{\mathrm{rel}}^{\mathrm{m}-\mathrm{R}, \mathrm{OH}^{-}}} \frac{Q_{\mathrm{m}-\mathrm{TS} 1}(T)}{Q_{\mathrm{m}-\mathrm{R}}(T) Q_{\mathrm{OH}^{-}}} \exp \left[-V^{\ddagger} / k_{\mathrm{B}} T\right]$

$k_{\mathrm{m}-2}(T)=\frac{k_{B} T}{h} \frac{1}{\Phi_{\mathrm{rel}}^{\mathrm{m}-\mathrm{R}, \mathrm{OH}^{-}}} \frac{Q_{\mathrm{m}-\mathrm{TS} 2}(T)}{Q_{\mathrm{m}-\mathrm{R}}(T) Q_{\mathrm{OH}^{-}}} \exp \left[-V^{\ddagger} / k_{\mathrm{B}} T\right]$ 
where $Q_{\mathrm{m}-\mathrm{R}}(T)$ is the reactant partition function for mesotartaric acid. Once again, we may take advantage of the mirror images to find that $Q_{\mathrm{m}-\mathrm{TS} 1}(T)=Q_{\mathrm{m}-\mathrm{TS} 2}(T)$, and therefore $k_{\mathrm{m}-1}(T)=k_{\mathrm{m}-2}(T)$.

For tartaric acid, there are three possible reactants that can form four possible products. Each product is only accessible from a single reactant. The properties of mirror images can be used to decrease the number of quantities that must be calculated. It is advisable not to employ any additional shortcuts when dealing with a complicated system like this one. We recommend for the rate constants to be used exclusively with their corresponding reactants and products and not to use formulas like Eq. (17) in any case where it may be conceptually difficult.

\section{Summary}

The present article shows how to calculate the rotational symmetry numbers for various molecular configurations and how to apply these symmetry numbers to transition state theory. For most reactions, the overall symmetry number is given by the ratio of the reactant and transition state rotational symmetry numbers, as given by Eq. (4). For bimolecular reactions where the two reactants are equivalent, the symmetry number is a rotational-translational symmetry number, which gives rise to an additional factor of two.

Section 3 contains several examples for calculating the symmetry number for reaction rate constants. Section 3.1 gives examples for symmetry numbers that are both intuitive and nonintuitive. Section 3.2 illustrates that Eq. (4) is technically valid for symmetric reactions, but the number of interest is typically twice as large as predicted by Eq. (4) because both the forward and reverse flux contribute equally. Section 3.3 illustrates how to account for chiral isomers using the symmetry number. Multiple conformers for reactants and/or transition states are illustrated in Sect. 3.4, and Sect. 3.5 deals with internal rotation, where the treatment used in some previous examples is improved upon. Section 3.6 gives an example involving an achiral diasteromer.

Many of the typical problems that arise when using symmetry numbers have been highlighted. Even complicated scenarios are treated systematically by properly calculating rotational symmetry numbers and differentiating between distinguishable and indistinguishable reaction paths.
Acknowledgments A.F.-R. thanks the Ministerio de Educación y Ciencia (MEC) and Xunta de Galicia (through the Dirección Xeral de Promoción Científica e tenolóxica do SUG) for financial support under projects \#BQU2003-01639 and \#2006/XA088, respectively. R. M.-P. thanks the MEC for a research contract under project \#BQU200301639. B.A.E. and D.G.T. thank the US Department of Energy for research support, through grant no. DOE-FG02-86ER13579.

\section{References}

1. Eyring H (1935) J Chem Phys 3: 107-115

2. Evans MG, Polanyi M (1935) Trans Faraday Soc 31: 875-894

3. Truhlar GG, Isaacson AD, Garrett BC (1985) Theory of chemical reaction dynamics. In: Baer M (ed) CRC Press, Boca Raton, vol 4, pp 65-137

4. Truhlar DG, Garrett BC, Klippenstein SJ (1996) J Phys Chem 100: 12771-12800

5. Fernández-Ramos A, Ellingson BA, Garrett BC, Truhlar DG (2007) Rev Comp Chem 23: 125-232

6. Garrett BC, Truhlar DG (1998) Encyclopedia of computational chemistry. In: Schleyer PvR, Allinger NL, Clark T, Gasteiger J, Kollman PA, Schaefer III HF (eds) Wiley, Chichester, vol. 5, pp. 3094-3104

7. Pollak E, Pechukas PJ (1978) J Am Chem Soc 100: 2984-2991

8. Coulson DR (1978) J Am Chem Soc 100: 2992-2996

9. Herzberg G (1950) Molecular spectra and molecular structure: spectra of diatomic molecules, 2nd edn, Van Nostrand D (ed) Princeton, NJ, pp. 132-139

10. McQuarrie DA (2000) Statistical mechanics. University Science Books, Sausalito

11. Wilson EB Jr (1935) J Chem Phys 3: 276-85

12. Bunker P, Jensen P (1998) Molecular symmetry and spectroscopy. Edited by NRC Research press, 2nd edn

13. Chakraborty A, Truhlar DG, Bowman JM, Carter S (2004) J Chem Phys 121: 2071-2084

14. Truhlar DG (1976) J Chem Phys 65: 1008-1010

15. Dennison DM (1927) Proc Roy Soc Lond Ser A 115: 483-486

16. Schatz GC, Kuppermann A (1976) J Chem Phys 65: 4668-4692

17. Truhlar DG, Abdallah J Jr (1974) Phys Rev A 9: 297-300

18. Hu W-P, Rossi I, Corchado JC, Truhlar DG (1997) J Phys Chem A 101: 6911-6921

19. Truhlar DG (1991) J Comput Chem 12: 266-270

20. Chuang YY, Radhakrishnan ML, Fast PL, Cramer CJ, Truhlar DG (1999) J Phys Chem A 103: 4893-4909

21. Chuang YY, Truhlar DG (2000) J Chem Phys 112: 1221-1228; (2004) 121: 7036(E); (2006) 124: 179903(E)

22. Ellingson BA, Lynch VA, Mielke SL, Truhlar DG (2006) J Chem Phys 125: 84305-84317

23. http://www.en.wikipedia.org/wiki/Tartaric_acid

24. http://www.en.wikipedia.org/wiki/Diastereomer 